\title{
Intrauterine device insertion-related complications: can they be predicted?
}

\author{
Miranda Farmer, MRCGP, MFFP, CMO, East Cheshire NHS Trust, Macclesfield District General Hospital, Victoria Road, \\ Macclesfield, UK; Anne Webb, MrCOG, MFFP, Consultant in Family Planning and Reproductive Health Care, Abacus \\ Centres for Contraception and Reproductive Health, North Liverpool Primary Care Trust, Liverpool, UK
}

Correspondence: Dr Miranda Farmer, Family Planning Department, Macclesfield District General Hospital, Victoria Road, Macclesfield SK10 3BL, UK. E-mail: mataylor@onetel.net.uk

(Accepted 20 May 2003)

Journal of Family Planning and Reproductive Health Care 2003; 29(4): 227-231

\begin{abstract}
Objective. To determine risk factors affecting the incidence of intrauterine device (IUD) insertion-related complications and failures and, in particular, whether postcoital IUD insertions have a higher incidence of complications than routine IUD insertions.

Design. Retrospective case notes analysis.

Participants. The study examined 545 case notes of patients having IUD insertions at East Cheshire NHS Trust family planning clinics between 1 October 1997 and 31 December 2000.

Main outcome measures. The incidence of complications at insertion, or up to 12 weeks after insertion, was determined and included failed insertion, cervical problems, syncope, bradycardia, convulsions, early perforation and early expulsion. Fourteen potential risk factors were examined to determine the effect on incidence of complications.

Results. Failed insertions were statistically more likely in women who had never previously had a vaginal delivery and also when a less experienced doctor performed the insertion. Nulliparous women were at statistically increased risk of cervical problems and bradycardia. Cervical problems at insertion also increased significantly with age. Patients who were amenorrhoeic at insertion were more likely to suffer an early IUD expulsion.

Conclusions. Of the potential risk factors, nulliparity was the most important. IUD insertion failures and complications were no more common in postcoital than routine IUD insertions. In general, complications were unpredictable, indicating the need for constant vigilance and the inserting doctor being trained and prepared to deal with any complication arising.
\end{abstract}

\section{Key message points}

- In general, intrauterine device (IUD) insertion-related complications are unpredictable.

- Doctors performing IUD insertions need to regularly update and maintain their skills in order to manage complications.

- Nulliparous women are at increased risk of complications, particularly bradycardia and cervical problems

- Postcoital IUD insertions are not in themselves associated with an increased risk of complications.

\section{Introduction}

As a result of a critical event experienced during a postcoital intrauterine device (IUD) insertion procedure, this study was undertaken to see whether lessons could be learned and applied in future practice in order to prevent further events. If insertions at high risk of complications can be predicted and risk factors for these insertions identified, then it may be possible to reduce the incidence of problems at insertion or at least be prepared for a possible adverse event.

One hypothesis is that because of patient anxiety after unprotected intercourse, postcoital insertions have an increased incidence of complications or failure. The alternative hypothesis, namely that there is no difference between complication incidence in postcoital and routine insertions, is also a possibility. It may be that a higher proportion of patients having postcoital insertions are nulliparous and that this increases the incidence of insertion problems. If IUD insertion complications cannot be predicted then there needs to be constant awareness of this possibility and updating of resuscitation techniques.

A MEDLINE search from 1966 to 2001 revealed few recent studies on IUD insertion failures and complications and the data quite often did not relate to modern IUDs in use today. Some risk factors for IUD insertion complications and failure have already been identified. However, whether an insertion is postcoital (as opposed to routine) had not been studied as a risk factor in the studies identified.

The insertion-related complications of interest in this study included insertion failure, problems negotiating the cervix, syncope, bradycardia, convulsions, perforation and expulsion.

From the literature search one author identified that the incidence of IUD insertion failure was between 2.3 and 8.3 per 1000 insertions, ${ }^{1,2}$ and pain during the insertion procedure was associated with increased likelihood of IUD insertion failure. However, as this is a concomitant event it cannot be used to predict patients at high risk of an insertion failure.

Risk factors for cervical spasm have not been studied directly. However, nulliparity is a risk factor for requiring cervical dilatation, and breastfeeding women are less likely to require cervical dilatation. ${ }^{3}$

Syncope has also been studied, revealing an incidence at IUD insertion of $2.1 \% .^{4}$ The risk factors identified from previous studies are moderate to severe pain at insertion ${ }^{4}$ and nulliparity. ${ }^{5}$

Electrocardiographic changes induced by Dalkon ${ }^{\circledR}$ shield insertion have been studied in one series of 25 women of whom 24 were nulliparous. This study reported an incidence of sinus bradycardia $(<60 \mathrm{bpm})$ of $32 \% .6$ Transient arrhythmias were noted in two patients. Another study has shown that the incidence of bradycardia is dependent on IUD type, with large and stiff IUDs associated with a higher frequency of bradycardia. $^{7}$ 
Convulsions are a rare complication of IUD insertion and have been estimated to have an incidence of 1 in 2000 insertions. ${ }^{8}$ No studies on risk factors for convulsions at insertion were identified by the literature search. However, in a report on three cases of convulsion at IUD insertion ${ }^{8}$ it was noted that none of the patients had previously had seizures but they all had a history of fainting in response to painful or emotional stimuli.

The incidence of perforation is estimated as being between 1.9 and 3.6 per 1000 insertions $^{9}$ and a previous Caesarean section has been identified as a risk factor. Other studies on the risk of uterine perforation show an increased risk in women who are breastfeeding at the time of IUD insertion. ${ }^{10,11}$ IUD design is thought to affect perforation rates, however the effect is difficult to establish as reports of direct comparisons are limited.

The literature review identified one study showing a higher risk of expulsion when the IUD was fitted during menstruation. ${ }^{12}$ Differences in expulsion rates due to IUD design are difficult to establish due to limited comparative trials.

This study therefore aimed to identify the incidence of insertion failure and complications and any patient, doctor or procedural risk factors for these events. The particular question of interest was whether postcoital IUD insertions have a higher incidence of complications than routine IUD insertions.

\section{Method}

The study covered all IUD insertions performed in East Cheshire NHS Trust family planning clinics (FPCs) between 1 October 1997 and 31 December 2000 inclusive. Patients were identified by a computer search. In addition, all the FPC appointment books were manually searched to pick up patients missed by the computer search. Each doctor working in the clinics was also asked whether they recorded IUD insertions (for recertification purposes) and these lists were examined to identify patients omitted by the computer search. In total 548 IUD insertions were identified.

For each insertion the medical records were examined to identify whether the insertion was successful or not and whether any complications occurred during insertion. Information on the following complications was specifically sought: insertion failure, cervical problems, syncope, bradycardia $(<60 \mathrm{bpm})$ and convulsions.

Perforation and expulsion occurring before the first routine follow-up appointment were also recorded. For most patients this was usually at 6-8 weeks after the insertion. Follow-up after 12 weeks was not included in this study.

Data were also completed from the patient record about the following possible risk factors: age of patient, postcoital versus routine insertion, same appointment versus elective IUD insertion, IUD type, whether the IUD was being changed, day of cycle on which insertion took place, parity, outcome of last pregnancy (vaginal delivery versus Caesarean section), whether the patient had ever had a vaginal delivery, breastfeeding status, past history of cone biopsy, past history of convulsions or epilepsy, analgesia used (none/lignocaine gel/paracervical block) and experience of doctor.

Same-visit insertions included insertions done at the time of presentation for an IUD (i.e. without an insertion appointment planned in advance). Elective insertions were defined as those insertions where the patient had an initial appointment for assessment and counselling about an IUD and returned for a separate appointment for the IUD insertion.

Doctors working in the service were asked when they obtained their Letter of Competence (LoC) in Intrauterine
Table 1 Complications occurring during 545 intrauterine device insertions

\begin{tabular}{lr}
\hline Complication & $\mathrm{n}(\%)$ \\
\hline Failure to insert successfully & $48(8.8)$ \\
Cervical problems & $63(11.5)$ \\
Syncope & $1 \quad(0.2)$ \\
Bradycardia $(<60 \mathrm{bpm})$ & $10(1.8)$ \\
Convulsions & 0 \\
\hline
\end{tabular}

Techniques or when they started inserting IUDs, and were asked to provide an estimate of how many IUDs they inserted per year. Experience was categorised into three groups:

- Experienced: $>5$ years post-LoC or equivalent and inserting $>12$ IUDs/year

- Limited experience: $<5$ years post-LoC and inserting $>12$ IUDs/year

- Inexperienced: $<5$ years post-LoC and/or inserting $<12$ IUDs/year.

The data were analysed using multiple logistic regression analysis in order to attempt to control for potentially confounding variables. Statistical advice was sought on numbers needed to obtain meaningful results. To detect a $5 \%$ difference in rates of complications with $80 \%$ statistical power 384-2740 cases needed to be studied. However, for complications with a very low incidence (i.e. syncope, convulsions, perforation) greater numbers would need to be studied.

\section{Results}

Data on 548 IUD insertions between 1 October 1997 and 31 December 2000 inclusive were studied. Three $(0.5 \%)$ sets of case notes could not be traced. Follow-up data up to 3 months was available for 407/497 successful insertion events $(82 \%)$. The average age of patients having IUD insertions was 33 years (range 15-49 years).

Seventy-two (13\%) insertions were postcoital. Of these, $28(38 \%)$ patients were nulliparous. Routine insertions were performed in $473(87 \%)$ patients and of these 75 (15\%) patients were nulliparous. Amongst the routine insertions, 128 were of the intrauterine system (IUS).

Complications occurring at, and up to 3 months after, insertion were recorded and are summarised in Tables 1 and 2 .

\section{Insertion failure}

Forty-eight insertion attempts failed $(8.8 \%)$ at the initial attempt. Sixteen patients had a failed initial insertion followed by a successful insertion with an alternative IUD at the same appointment. This leaves 32 'true' insertion failures $(5.9 \%)$.

There were a variety of reasons for the insertion failures as shown in Figure 1. Procedural problems resulting in failure to fit the IUD included:

- inability to sound the uterus because of acute anteversion and anteflexion,

- bending of the introducer tube, or

- removal because of uncertainty over the position of an IUD during a training session.

Logistic regression analysis showed that patients with a

Table 2 Complications occurring up to 3 months after $416^{a}$ intrauterine device insertions

\begin{tabular}{lr}
\hline Complication & $\mathrm{n}(\%)$ \\
\hline Perforation & $1(0.2)$ \\
Expulsion & $24(5.8)$ \\
\hline
\end{tabular}

ancludes 407 successful insertions and nine immediate expulsions. 
Table 3 Odds ratios (and 95\% CI) for failure to insert according to doctor experience

\begin{tabular}{lccl}
\hline Doctor experience & Cases (n) & Failures (n) & Odds ratio (95\% CI) \\
\hline Experienced & 436 & 29 & 1.0 \\
Limited experience & 34 & 4 & $1.9(0.6-5.7)$ \\
Inexperienced & 72 & 14 & $3.0(1.5-6.2)$ \\
\hline
\end{tabular}

previous history of vaginal delivery were at lower risk of insertion failure $(\mathrm{p}=0.039)$.

IUD insertions performed by less experienced doctors had a statistically significant higher risk of insertion failure (Table 3).

There was no statistically significant increased risk of IUD insertion failure in postcoital, as compared to routine, insertions. Excluding insertions of the IUS, the failure rate for postcoital (copper) IUDs was $9.7 \%$ and for routine copper IUDs it was $9.9 \%$.

There was no statistical difference in risk of insertion failure for any of the other risk factors studied.

\section{Cervical problems}

In total there were 63 cases $(11.5 \%)$ with cervical problems. In five cases there was initial difficulty passing the sound through the cervix; the remainder were problems passing the IUD. Of these, 37 episodes were overcome during the initial insertion attempt (59\%) and a further 11 insertions were successful at a second appointment.

There were 15 events remaining where IUD insertion was unsuccessful, giving an incidence of 'true' cervical problems of $2.8 \%$.

Logistic regression revealed a statistically significant increased risk of cervical problems with age $(p=0.01)$. This effect was more obvious in nulliparous patients than in multiparous women (Figure 2).

Patients who had ever had a vaginal delivery were significantly less likely to develop cervical spasm than patients with no prior history. The odds ratio for cervical problems occurring in patients who had had a previous vaginal delivery compared with those who had not was 0.4 (95\% CI 0.2-0.9).

The results showed no statistically significant increased risk of cervical problems in postcoital insertions or with the other risk factors studied.

\section{Syncope}

Syncope affected one patient $(0.2 \%)$ and so no statistical analysis was possible.

\section{Bradycardia}

There were 10 episodes of bradycardia giving an incidence of $1.8 \%$. Of the 10 patients, nine were nulliparous. Of the nulliparous patients, $8.7 \%$ developed a bradycardia

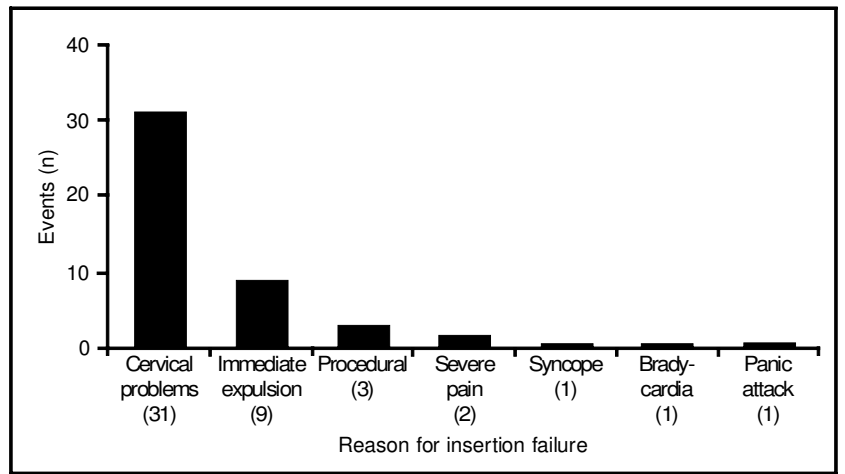

Figure 1 Number of and reasons for initial intrauterine device insertion failure

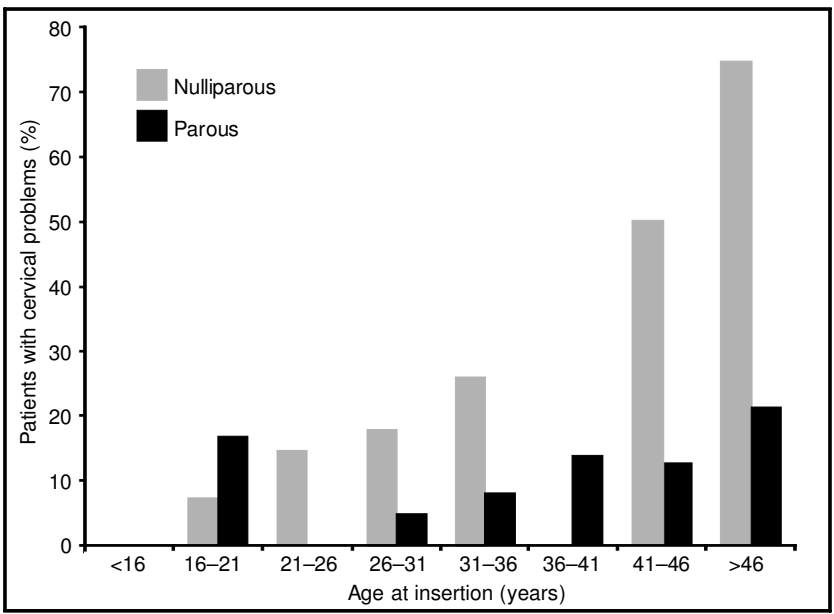

Figure 2 Proportion of patients with cervical problems according to age and parity

compared to $0.2 \%$ of parous patients. The odds ratio of nulliparous women developing a bradycardia compared to parous women was 18.5 (95\% CI 3.9-88.3). Apart from nulliparity, none of the other risk factors including postcoital insertion were associated with bradycardia.

\section{Convulsions}

There were no episodes of convulsion at IUD insertion during the period studied.

\section{Perforation}

There was one perforation discovered at 6 weeks postinsertion $(0.2 \%)$ and risk factor analysis was not possible.

\section{Expulsion}

There were 24 expulsions in total $(5.8 \%)$, of which nine occurred immediately and 15 were discovered before 12 weeks' postinsertion. Amenorrhoeic patients whose last menstrual period was at least 6 weeks prior to insertion were statistically more likely to have an IUD expulsion ( $p$ $=0.021$ ). All of these patients had previously used DepoProvera $^{\circledR}$ (none were breastfeeding). The odds ratio for expulsion in the amenorrhoeic patients was 2.9 (95\% CI 1.2-7.2).

The results showed that there was no statistically significant increased risk of IUD insertion-related complications in postcoital, compared to routine, insertions.

There was no statistical difference in risk of insertion failure for any of the other risk factors studied.

\section{Discussion}

\section{Insertion failure}

The main reasons for insertion failure in the current study were problems passing the sound or IUD through the cervix and immediate expulsion.

This study identified two significant risk factors for insertion failures. The first important factor was that those patients with a previous history of vaginal delivery were at lower risk of insertion failure. A prior history of vaginal delivery was also associated with a lower incidence of other complications (e.g. cervical problems and bradycardia) and this was therefore reflected in the lower overall insertion failure rate. This could be due to the fact that patients with a past history of vaginal delivery have a slightly wider cervical canal than nulliparous women in whom the cervical canal has never been stretched.

The second important factor when looking at insertion failure was the experience of the doctor inserting the IUD. 
The results showed a clear decrease in failure rate with experience. Those doctors with limited experience had twice the failure rate of experienced doctors and those doctors who were inexperienced had three times the failure rate.

The main reason for this study being conducted was to compare insertion failures and complications in postcoital versus routine insertions. With regard to insertion failures, from the results of this study there was no statistically significant difference between these groups.

One hypothesis was that nulliparous women may be over-represented in the postcoital group and that this could be the main reason for insertion problems. Indeed the postcoital group was made up of $38 \%$ nulliparous women compared to $15 \%$ in the routine insertion group. However, parity was not statistically significant as a risk factor. As has been discussed, a history of previous vaginal delivery was more important as a factor overall than simply parity. The results of this study indicate that the null hypothesis is likely to be true, namely that there is no difference in insertion failure rates between those having postcoital versus routine insertions.

\section{Cervical problems}

The higher rate of cervical problems related to age, especially in nulliparous women, may be due to the cervix becoming less elastic with age and also less ready to dilate because of an increased proportion of fibrous tissue. In parous women the effect of ageing is still apparent but there seem to be fewer cervical difficulties at IUD insertion. This is possibly because the cervix has been stretched and weakened by childbearing, resulting in the cervical canal being more able to accommodate the uterine sound or IUD.

\section{Bradycardia}

Parity was an important risk factor, with nulliparous women being at 18 times higher risk of developing a bradycardia compared to parous women. However, the estimate of the magnitude of the increase needs to be interpreted with caution, as there were wide confidence intervals for this result. Again tying in with cervical problems, this would tend to indicate that, an inelastic, 'never-dilated' cervix predisposes to complications. In the case of bradycardia, this may be mediated by more intense cervical manipulation to overcome a resistant cervix resulting in increased vagal stimulation.

\section{Expulsion}

The amenorrhoeic women were almost three times as likely to have an early expulsion compared to the menstruating women in this study. Of the amenorrhoeic women who expelled their IUD, all had previously been using Depo-Provera (none were breastfeeding). DepoProvera thins the endometrium and may lead to lower oestrogen levels, which could slightly reduce the uterine cavity and make the insertion more difficult. The uterus is known to vary in shape and tone throughout the menstrual cycle affecting the uterine cavity length versus cervical length. ${ }^{13}$ In prolonged amenorrhoea the uterine cavity length may be much reduced (even if the uterine sound length remains unchanged) resulting in dimensional incompatibility with the IUD, which is then expelled. However, only one-third of the expulsion cases occurred in amenorrhoeic women and obviously other factors must come into play.

The finding is also contrary to a previously reported study showing a higher expulsion rate in IUDs fitted during menstruation. ${ }^{12}$ However, women with prolonged amenorrhoea were excluded from the study group which only included patients if their cycle day of insertion, and therefore their last menstrual period, was known. The difference in findings may be because there are two subgroups of women: those menstruating in whom there is increased uterine contractile activity such that the IUD is expelled together with menstrual blood; and those with prolonged amenorrhoea who are more susceptible to IUD expulsion due to mismatch of uterine and IUD dimensions.

\section{Study limitations}

The main limitation of the study was collecting data on sufficient numbers of cases. The number of cases studied was sufficient for complications such as insertion failure and cervical problems. However, for complications with a very low incidence (i.e. syncope, convulsions, perforation) far greater numbers needed to be studied. This limitation is reflected in the wide confidence intervals for the results. This means that caution must be applied in interpreting the results, and ideally a large-scale study is needed. Each variable with its confidence intervals was calculated and recorded in the dissertation for which this work was originally carried out. ${ }^{14}$

Another limitation of this study is the fact that it was retrospective, thus allowing potential for observer bias. The greatest difficulties were legibility of the case notes, incomplete records and categorisation of complications and risk factors. However, from knowledge of the staff involved, it is felt likely that most significant problems would have been recorded. A large, prospective study would limit this problem.

\section{Conclusions}

Overall it appears that IUD insertion-related complications are related to anatomical and physiological factors in addition to the experience of the doctor inserting the IUD. If any factor is predictive then nulliparous women seem to experience a higher incidence of IUD insertion-related complications, especially cervical problems and bradycardias. This also applies to women who have only ever had Caesarean sections, and a past history of ever having had a vaginal delivery is associated with a lower incidence of insertion problems.

The results of this study indicate that postcoital IUD insertions in themselves are not associated with any increased risk of insertion failure or complications.

Extra care is needed with nulliparous women, but there is no guarantee that problems will not arise at any time. Consequently at all IUD insertions the doctor needs to be vigilant and constantly updated in resuscitation techniques and the management of cervical shock. The insertion should always take place in appropriate surroundings with adequate and trained support staff present and resuscitation equipment available.

Although limited by small numbers for the rare insertion-related complications, this study highlights an important area for future research on a larger scale. This study also studied all IUD insertions (including the IUS) and therefore in order to clarify the position with regard to postcoital as opposed to routine IUD insertions a larger study on copper IUDs is needed.

\footnotetext{
Acknowledgements

The authors would like to thank Dr Tony Scallan at the Department of Computing and Mathematics at Manchester Metropolitan University for his advice on statistics. The authors are also grateful to the clerical and clinical staff at East Cheshire family planning clinics for all their help with this study.
}

Statements on funding and competing interests

Funding. None identified.

Competing interests. None identified. 
References

1 Chi I-C, Wilkens LR, Siemens AJ, et al. Rare events at insertion of an intrauterine device - a review of an international experience. $A d v$ Contracept 1987; 3: 49-61.

2 Chi I-C, Rogers S. Failure to insert an intrauterine device. Contracept Deliv Syst 1983; 4: 207-211.

3 Chi I-C, Champion CB, Wilkens LR. Cervical dilatation in interval insertion of an IUD. Who requires it and does it lead to a high expulsion rate? Contraception 1987; 36: 403-415.

4 Chi I-C, Wilkens LR, Siemens AJ, et al. Syncope and other vasovagal reactions at interval insertion of Lippes Loop D - who is most vulnerable? Contraception 1986; 33: 179-187.

5 Conrad CC, Ghazi M, Kitay DZ. Acute neurovascular sequelae of intrauterine device insertion or removal. J Reprod Med 1973; 11: 211-212.

6 Sherrod DB, Nicholl W. Electrocardiographic changes during intrauterine contraceptive device insertion. Am J Obstet Gynecol 1974; 119: 1044-1051.

7 Aznar R, Reynoso L, Ley E, et al. Electrocardiographic changes induced by insertion of an intrauterine device and other uterine manipulations. Fertil Steril 1976; 27: 92-96.

8 Faden A, Spire JP, Faden R. Fits, faints and the IUD. Ann Neurol 1977; 1: 305-306.

9 Chi I-C, Feldblum PJ, Rogers SM. IUD-related uterine perforation: an epidemiologic analysis of a rare event using an international dataset. Contracept Deliv Syst 1984; 5: 123-130.

10 Heartwell SF, Schlesselman S. Risk of uterine perforation among users of intra-uterine devices. Obstet Gynecol 1983; 61: 31-36.

11 Andersson K, Ryde-Blomqvist E, Lindell K, et al. Perforations with intrauterine devices - report from a Swedish survey. Contraception 1998; 57: 251-255.

12 White MK, Ory HW, Rooks JB, et al. Intrauterine device termination rates and the menstrual cycle day of insertion. Obstet Gynecol 1980; 55: $220-224$.

13 Hasson H. Uterine geometry and IUCD design. Br J Obstet Gynaecol 1982; 89: $1-10$.

14 Farmer M. IUD insertion related complications - can they be predicted? Dissertation accepted for MFFP, 2001

\section{S UPPORT SERVICES FOR EVRA}

Janssen-Cilag Ltd has started a free text message services to remind Evra users when they need to replace their patch or start the patch-free week. This service is available via a new website for women on Evra, and there is also a separate Evra website for healthcare professionals.

The password-protected website www.evra.co.uk includes comprehensive information for Evra users and details of the Evra Users' Support Line telephone service 08458501601

Healthcare professionals access information and resources via their own password-protected website, www.evrapatch.co.uk, which contains clinical information about Evra, downloadable educational materials and an online ordering service for health professional and Evra-user resources.

JANSSEN-CILAG Ltd
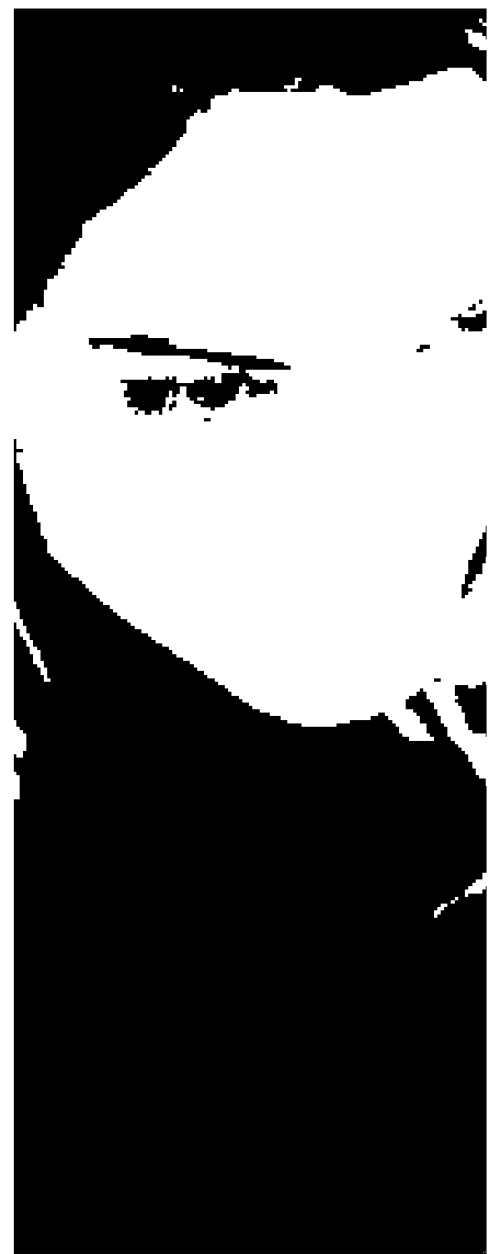

The sites complement the webs ite, www.evriwoman.co.uk that gives general information on contraception and sexual health. 